\title{
Cascaded Multicycle Terahertz-Driven Ultrafast Electron Acceleration and Manipulation
}

\author{
Dongfang Zhang $\odot,{ }^{1, *}$ Moein Fakhari, ${ }^{1,2}$ Huseyin Cankaya, ${ }^{1,2}$ Anne-Laure Calendron, ${ }^{1}$ \\ Nicholas H. Matlis, ${ }^{1}$ and Franz X. Kärtner ${ }^{1,2}$ \\ ${ }^{1}$ Center for Free-Electron Laser Science, Deutsches Elektronen Synchrotron, \\ Notkestrasse 85, 22607 Hamburg, Germany \\ ${ }^{2}$ Department of Physics and The Hamburg Centre for Ultrafast Imaging, University of Hamburg, \\ Luruper Chaussee 149, 22761 Hamburg, Germany
}

(Received 5 July 2019; revised manuscript received 25 October 2019; accepted 6 February 2020; published 19 March 2020)

\begin{abstract}
Terahertz (THz)-based electron acceleration and manipulation has recently been shown to be feasible and to hold tremendous promise as a technology for the development of next-generation, compact electron sources. Previous work has concentrated on structures powered transversely by short, single-cycle $\mathrm{THz}$ pulses, with millimeter-scale, segmented interaction regions that are ideal for acceleration of electrons in the sub- to few-MeV range, where electron velocities vary significantly. However, in order to extend this technology to the multi-MeV range, an investigation of approaches supporting longer interaction lengths is needed. Here, we demonstrate first steps in electron acceleration and manipulation using dielectrically lined waveguides powered by temporally long, narrow-band, multicycle $\mathrm{THz}$ pulses that copropagate with the electrons. This geometry offers centimeter-scale single-stage interaction lengths and offers the opportunity to further increase interaction lengths by cascading acceleration stages that recycle the $\mathrm{THz}$ energy and rephase the interaction. We prove the feasibility of $\mathrm{THz}$-energy recycling for the first time by demonstrating acceleration, compression, and focusing in two sequential $\mathrm{Al}_{2} \mathrm{O}_{3}$-based dielectric capillary stages powered by the same multicycle $\mathrm{THz}$ pulse. Since the multicycle THz energy achievable using laser-based sources is currently a limiting factor for the maximum electron acceleration, recycling the $\mathrm{THz}$ pulses provides a key factor for reaching relativistic energies with existing sources and paves the way for applications in future ultrafast electron diffraction and free-electron lasers.
\end{abstract}

DOI: 10.1103/PhysRevX.10.011067

Subject Areas: Optics, Photonics

\section{INTRODUCTION}

Femtosecond-duration relativistic electron beams are in demand for a wide range of applications from ultrafast electron diffraction [1,2] and microscopy [3] to ultrafast $\mathrm{x}$-ray sources [4]. Microwaves in the radio-frequency (rf) regime $(1-10 \mathrm{GHz})$ have been the conventional choice for accelerating electrons but face significant challenges in meeting the request for future acceleration and manipulation of particle beams on the femtosecond timescale. The limited acceleration fields sustainable at a low frequency also necessitate kilometer-scale facilities to reach highly relativistic beams, making these devices highly exclusive and costly. Therefore, there is a strong motivation for exploring alternative solutions which are more compact,

\footnotetext{
*To whom correspondence should be addressed. dongfang.zhang@cfel.de

Published by the American Physical Society under the terms of the Creative Commons Attribution 4.0 International license. Further distribution of this work must maintain attribution to the author(s) and the published article's title, journal citation, and DOI.
}

economic, and adapted for pushing the resolution frontiers. Novel laser-based accelerator concepts provide intrinsic optical synchronization, allow scaling to smaller accelerator structures, and generate substantially stronger fields for acceleration and beam manipulation. A prime example is laser-plasma accelerators (LPAs) [5,6], which can reach field gradients of more than $10 \mathrm{GV} / \mathrm{m}$ and have produced multi-GeV electron beams with a percent-level energy spread. However, LPAs need considerable laser development to operate at high repetition rates and are hampered by instabilities and difficulties in controlling beam parameters associated with the dynamical nature of the plasma. Dielectric laser accelerators can reach $\mathrm{GV} / \mathrm{m}$ accelerating fields $[7,8]$ but, however, present significant challenges in beam control and are so far limited to low bunch charges in the single-electron range due to their submicron-scale cross sections.

Recently, there has been significant interest in the use of terahertz (THz)-based particle acceleration and beam manipulation as a new approach with the potential to overcome certain limitations of $\mathrm{rf}$ accelerators and other laser-based concepts. In particular, the picosecond timescale of laser-generated $\mathrm{THz}$ sources is expected to enable 
order-of-magnitude increases in sustainable field strength $[9,10]$ to the $\mathrm{GV} / \mathrm{m}[11-14]$ range and increased precision in timing compared to $\mathrm{rf}$ sources while still supporting moderate charge at the picocoulomb level [15]. Proof-ofprinciple demonstrations with single-cycle $\mathrm{THz}$ pulses have resulted in multi-keV acceleration [16,17] and high-field manipulations of electron-bunch phase space [18-20] proving the feasibility of compact $\mathrm{THz}$-based devices for future radiation sources or as components for boosting the performance of existing accelerators as well as a promising solution to produce high-repetition, high-energy ultrafast electron bunches, which are highly desired in ultrafast electron diffraction [21,22]. However, the interaction lengths in this configuration are limited by available single-cycle pulse energies to the millimeter range. A $\mathrm{THz}$-driven inverse free-electron laser (IFEL) scheme has been proposed $[23,24]$ that provides a potential solution to solve the dispersion, dephasing, and walk-off problems for relativistic electron using single-cycle $\mathrm{THz}$ [24].

Copropagation designs powered by narrow-band multicycle $\mathrm{THz}$ radiation have the potential to improve the interaction length as well as the symmetry of the field distribution. In particular, dielectrically lined waveguides (DLWs) have attracted interest in the accelerator community for their versatility to accelerate, manipulate, and characterize $[25,26]$ electron beams. They require a simpler fabrication process using commercial solutions with percent-level precision [27] which enable tuning of the phase and group velocity of the THz wave inside the DLW. Proofof-principle experiments are performed by Nanni et al. [16] demonstrating the feasibility of THz-driven DLW accelerators. However, these experiments use single-cycle pulses of $10 \mu \mathrm{J}$ to modulate by $7 \mathrm{keV}$ the energy of electrons injected at $60 \mathrm{keV}$. The interaction length is limited to $3 \mathrm{~mm}$, which is due to the use of a short $\mathrm{THz}$ pulse and mismatch between the electrons and the $\mathrm{THz}$ phase front. Meanwhile, the injected long electron bunch leads to a large energy spread with not only acceleration but also deceleration at the same time. Dispersion, dephasing, and temporal walk-off are three critical effects which must be managed to achieve long interaction lengths. Dispersion results from the frequency dependence of the $\mathrm{THz}$ phase velocity in the waveguide and causes the pulse to temporally stretch and reduce in field strength; dephasing arises when the electrons slip from the accelerating to the decelerating phase of the field due to a difference between the electron velocity and the $\mathrm{THz}$ phase velocity; and "walk-off" arises when the electron bunch loses overlap with the $\mathrm{THz}$ pulse envelope due to a difference between the electron velocity and the $\mathrm{THz}$ group velocity. Dispersion can be mitigated by using $\mathrm{THz}$ sources of sufficiently narrow bandwidth which are now available with sub-mJ energies and subpercent bandwidths [28], due to recent developments in $\mathrm{THz}$ generation using periodically poled lithium niobate (PPLN) [29]. The use of narrow-band pulses can also lead to improved $\mathrm{THz}$ spatial mode quality [30], simplified beam transportation, and improved coupling efficiency into the waveguides. In addition, limited by the breakdown threshold of dielectric [31] compared with single-cycle $\mathrm{THz}$ using multicycle $\mathrm{THz}$ allows us to store more THz energy in the waveguide, which is very important for high-energy electron acceleration. Addressing dephasing and walk-off, however, requires the development of structures and methodologies beyond the simple DLW concept. Tapering of the DLW properties is proposed as one solution for addressing phase slippage [32]. However, this approach requires high injection energies and very tight tolerances on fabrication and has so far not been demonstrated. Meanwhile, as the conversion efficiency of current laser-based $\mathrm{THz}$ generation is still rather low $(<1 \%)$, it limits its further development as high-energy electron sources for a tabletop radiation source.

Here, we explore, for the first time, the use of narrowband $\mathrm{THz}$ pulses to drive a DLW and introduce a new scheme based on cascading of DLWs, which enables both rephasing and retiming of the electrons relative to the $\mathrm{THz}$ pulse, demonstrating reuse of the $\mathrm{THz}$ energy for the first time. Relative to previous work [16], we increase the interaction length by an order of magnitude to approximately $3 \mathrm{~cm}$. Using only about $20 \mathrm{~nJ}$ of THz pulse energy, we accelerate $53 \mathrm{keV}$ electrons by approximately $1.6 \mathrm{keV}$, which represents an approximately tenfold improvement in energy transfer between the $\mathrm{THz}$ and the electrons, compression of the electron bunch by a factor of 10 down to approximately $150 \mathrm{fs}$ (FWHM), and spatial focusing the electron beam by a factor of 3 .

\section{RESULTS}

\section{A. Experiment setup}

In the experimental setup (Fig. 1), a $53 \mathrm{keV}$ phototriggered dc gun is used as an injector for the multistage DLW-based electron accelerator and manipulator which is powered by narrow-band, multicycle $\mathrm{THz}$ pulses. The electron beam from the DLW device is analyzed by a segmented terahertz electron accelerator and manipulator (STEAM) device [20], which is used as a streak camera to measure the bunch length with a resolution of approximately $150 \mathrm{fs}$, and by a tunable electromagnetic dipole coupled with a microchannel plate, which is used to measure the energy with a resolution of approximately $1 \mathrm{keV}$ and deflection of the beam. Ultraviolet (UV) pulses for photoemission in the dc gun, multicycle THz pulses to drive the DLW device, and single-cycle THz to drive the STEAM device are all created using a single infrared $\mathrm{Yb}$ : KYW laser system producing 4-mJ, 650-fs (FWHM), $1030-\mathrm{nm}$ pulses at a $1 \mathrm{kHz}$ repetition rate [33]. The UV pulses are generated by two successive stages of second harmonic generation, 50-ps-long (FWHM) multicycle $\mathrm{THz}$ 


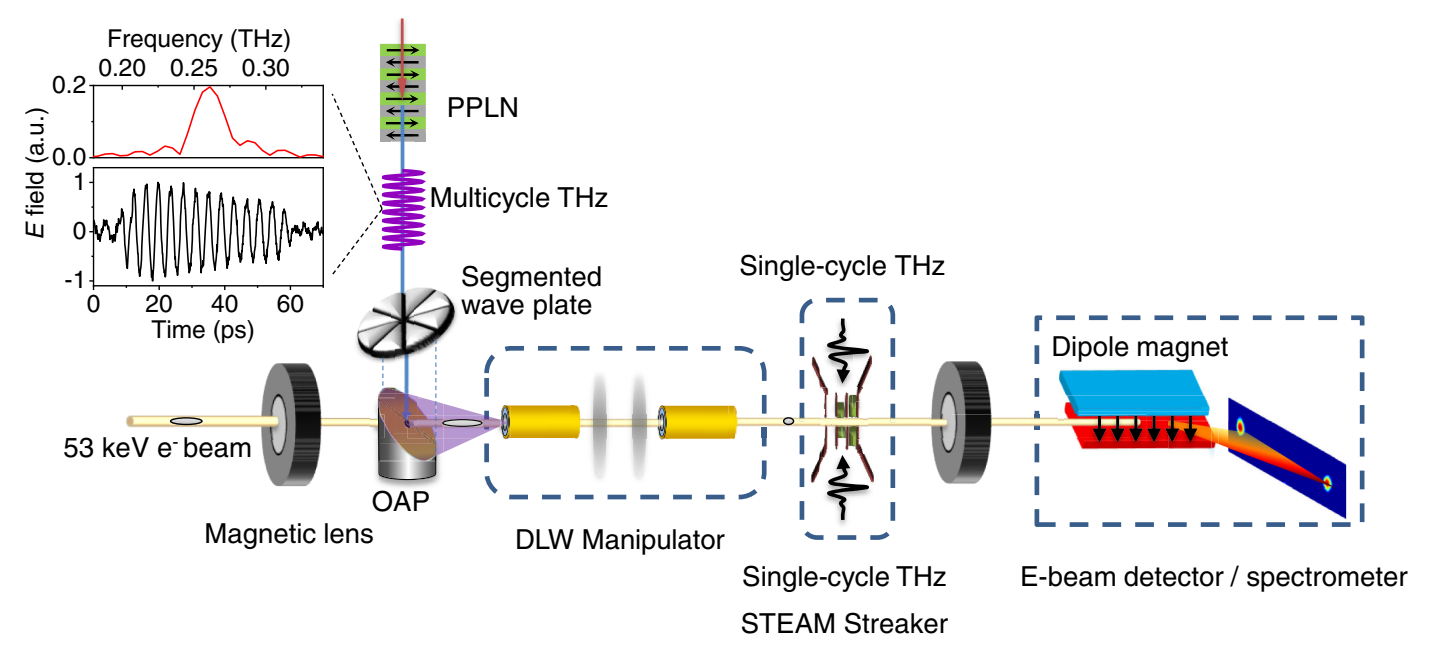

FIG. 1. Experimental setup. A small fraction of the 1030-nm infrared optical beam is converted to 257-nm based on two-stage second harmonic generation. The 257-nm UV pulse is directed onto a gold photocathode generating electron pulses, which are accelerated to $53 \mathrm{keV}$ by the dc electric field with around $1 \mathrm{fC}$ charge. The same infrared laser also drives a multicycle THz generation stage and two single-cycle THz stages for the DLW manipulator and the STEAM streaker, respectively.

pulses are generated by intraband difference frequency generation in a 5-mm-long MgO:PPLN crystal [34] (Fig. 1, inset), and single-cycle $\mathrm{THz}$ pulses are generated via the tilted-pulse-front method in a $\mathrm{LiNbO}_{3}$ prism [11]. The linearly polarized multicycle $\mathrm{THz}$ beam, which has 13 cycles and a center frequency of $0.26 \mathrm{THz}$, is then converted to a radially polarized beam via a segmented wave plate before being coupled into the DLW device collinearly to the electron motion using an off-axis-parabolic mirror and horn structure that concentrates the $\mathrm{THz}$ fields into the interaction zone.

The DLW design consists of a cylindrical copper waveguide of diameter $790 \mu \mathrm{m}$ and length $30 \mathrm{~mm}$ with a dielectric layer of alumina $\left(\mathrm{Al}_{2} \mathrm{O}_{3}, \mathrm{THz}\right.$ refractive index $n=3.25$; see Supplemental Material, Fig. S3 [35]) with a wall thickness of $140 \mu \mathrm{m}$. The waveguide supports a traveling transverse-magnetic waveguide mode $\left(\mathrm{TM}_{01}\right.$ mode), the axial component of which provides the longitudinal field for acceleration and deceleration (Fig. 2). The dimensions and index of the dielectric material are chosen from the calculated map shown in Fig. 2, providing a phase velocity of approximately $0.43 c$ at $0.26 \mathrm{THz}$ : These parameters match the initial velocity of the electrons and optimize the device for electron acceleration and manipulation functions. Alumina is also desirable as a dielectric material because of its transparency and high damage threshold. The dimensions and index of the DLW result in a group velocity of the $\mathrm{THz}$ pulse that is approximately $0.25 c$, which is significantly slower than the electron velocity and leads to a strong walk-off effect. The diameter of the vacuum core of the DLW is $510 \mu \mathrm{m}$, which represents the clear aperture for the electron propagation.

Conical horns are used to couple $\mathrm{THz}$ energy into and out of the waveguides. In the acceleration mode, $3.5 \mathrm{~mJ}$ of the $\mathrm{Yb}: \mathrm{KYW}$ laser pulse energy is used for multicycle $\mathrm{THz}$ generation, resulting in approximately $20 \mathrm{~nJ} \mathrm{THz}$ energy injected into the DLW via the horn coupler. For compression and focusing, $0.5-\mathrm{mJ}$ laser pulses are used for multicycle $\mathrm{THz}$ generation, and the rest $(3 \mathrm{~mJ})$ is used for single-cycle $\mathrm{THz}$ generation. The $6 \mu \mathrm{J}$ single-cycle pulses centered at $300 \mathrm{GHz}$ are injected into the STEAM streak camera [20], which has a time resolution of approximately $150 \mathrm{fs}$.

\section{B. Operation}

The function of the device is selected by tuning the relative delay between the $\mathrm{THz}$ pulse and the electron bunch which determines the phase of the $\mathrm{THz}$ waveform that the electron bunch experiences. There are four key phase points within the waveform corresponding to distinct behaviors: the positive and negative crests of the waves, where the field gradient is minimized, and the positive and negative "zero crossings" of the field, where the field gradient is maximized. The positive and negative crests correspond to deceleration and acceleration of the electron bunch, respectively, but leave the bunch spatial and temporal dimensions unchanged. At the zero crossings, by contrast, the average energy and transverse position of the bunch are unchanged, but the bunch experiences a combination of spatial and temporal reshaping. At the positive zero crossing, where the field gradient is positive, the bunch becomes stretched in time but focused in space, while at the negative zero crossing, the bunch is temporally compressed but expands in space. As described by the Panofsky-Wenzel theorem [37], the longitudinal gradient of the transverse force is proportional to the transverse gradient of the longitudinal force. 


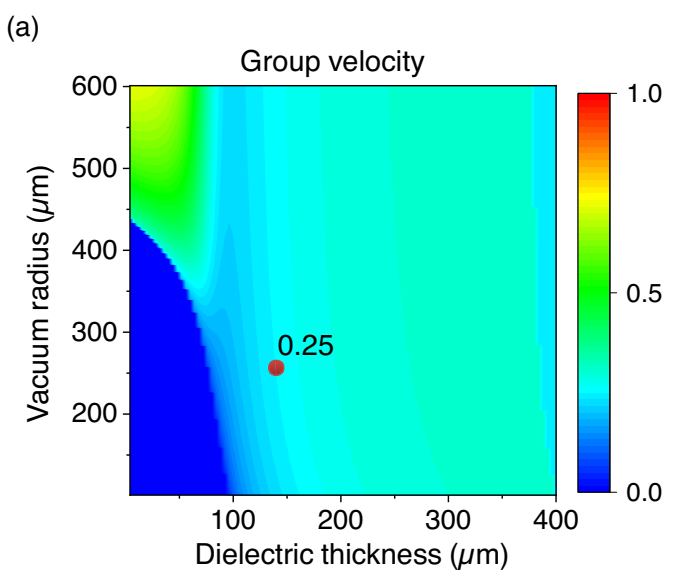

(c)

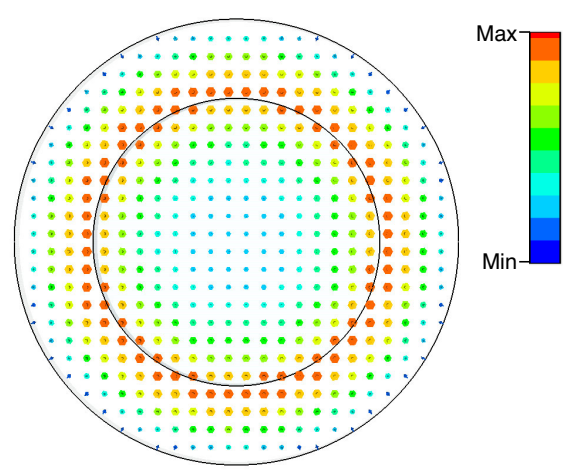

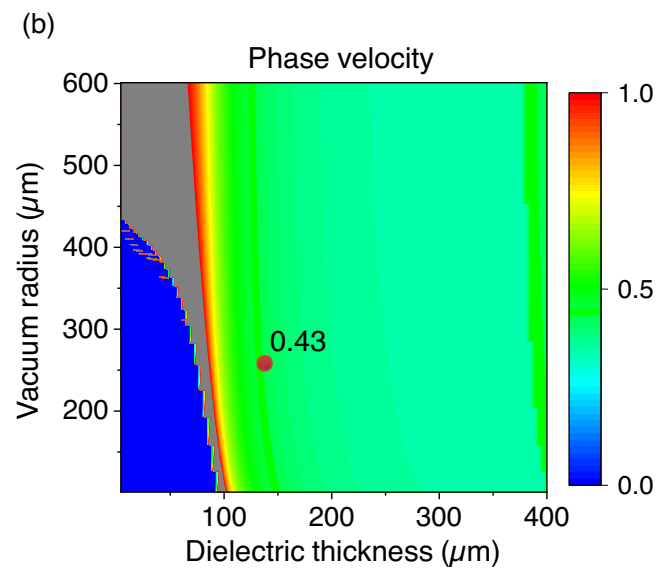

(d)

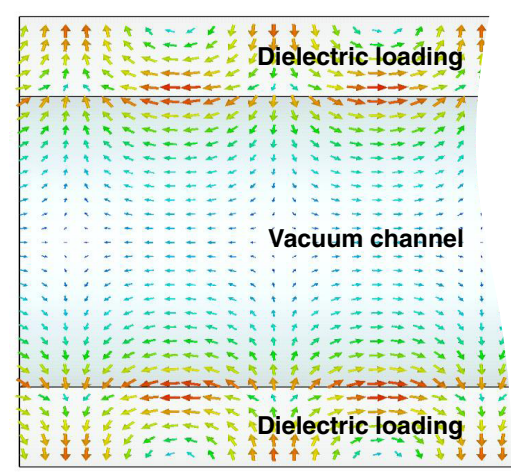

FIG. 2. $\mathrm{TM}_{01}$ - mode parameters of DLW. Group (a) and phase (b) velocity of the $\mathrm{TM}_{01}$ mode at $260 \mathrm{GHz}$ in a circular copper waveguide with dielectric loading. The red dots represent the design point for electron beam manipulation with $250 \mu \mathrm{m}$ vacuum radius and $140 \mu \mathrm{m}$ dielectric thickness. Transverse (c) and longitudinal (d) cross section of the electric field distribution at $\mathrm{TM}_{01}$ mode with $43 \%$ phase velocity at the design parameter for the experiment simulated with CST Microwave Studio [36]. The dielectric wall thickness is $140 \mu \mathrm{m}$, and the vacuum channel diameter is $510 \mu \mathrm{m}$.

To optimize the energy gain of a single DLW stage, the interaction length in the accelerating phase must be maximized, and the decelerating phase must be avoided. The interaction length can be maximized by initially setting the phase velocity to be larger than the injected electron speed by an amount just enough that the electrons can gain energy and start to overtake the phase velocity of the $\mathrm{THz}$ wave. To avoid the decelerating phase, two methods can be used. The first method relies on coupling the $\mathrm{THz}$ wave out of the DLW before electrons slip into the decelerating half-cycle. Another way is to take advantage of the walk-off between the $\mathrm{THz}$ pulse and the electron bunch by designing the $\mathrm{THz}$ to be short enough that electrons enter the decelerating half-cycle only after they have overtaken the envelope of the $\mathrm{THz}$ field. The second method ensures much cleaner acceleration, because the electrons do not interact with the less homogenous fields in the coupler region.

For compression and focusing, the speed of the electron bunch should be matched to the phase velocity of the $\mathrm{THz}$ pulse inside the waveguide, since neither changes during the interaction. The interaction length is then limited only by the THz pulse duration and the rate of walk-off. In order to minimize unwanted effects at the entrance and exit boundaries, it is desirable that the complete interaction occur inside the DLW. As long as the THz group velocity is less than the electron velocity, a clean THz-electron interaction can be achieved by choosing a waveguide of sufficient length so that the electrons have overtaken the $\mathrm{THz}$ pulse before exiting.

Compression of the electron bunch is based on "velocity bunching" [38]. In this technique, the electron bunches are placed at the negative zero crossing so that the tail experiences acceleration and the head experiences deceleration, but the bunch sees no average energy gain. The imparted momentum gradient then leads to compression of the bunch during propagation. For stretching, the bunch is placed at the positive zero crossing and experiences the opposite gradient.

The focusing and defocusing of the bunch which also occur at the zero crossings are due to the transverse electric fields. These fields are partially canceled by the transverse-deflection force from the magnetic field of the traveling THz.

In a circular waveguide, the net transverse force on an electron which is moving with velocity $v_{e}$ parallel to the $z$ component of the electric field [39] can be derived as (see Supplemental Material [35] for detailed information): 


$$
F_{\perp}=F_{e}+F_{m}=q\left(E_{r}-v_{e} B_{\varphi}\right) \propto q\left(1-\frac{v_{e} v_{\mathrm{ph}}}{c^{2}}\right)
$$

where $c$ is the speed of light, $v_{e}$ is the electron velocity, $v_{\mathrm{ph}}$ is the phase velocity of the electric field, $q$ is the charge of the electron, and $F_{e}$ and $F_{m}$ are the forces of the electric and magnetic components, respectively. As can be seen from the above equation, for the relativistic electrons whose velocity is close to $c$, the net transverse field tends to vanish. However, for nonrelativistic electrons or when $v_{\mathrm{ph}}$ or $v_{e}$ is less than $c$, there would be a net deflecting force acting on the electrons.

\section{Single-DLW results}

Figure 3 shows the acceleration results from a single DLW. As the delay between the electrons and the $\mathrm{THz}$ is varied, a cross-correlation between the $\mathrm{THz}$ and electron can be seen [Fig. 3(e)]. A maximum energy shift of around $1 \mathrm{keV}$ is observed using approximately $20 \mathrm{~nJ}$ multicycle THz pulse energy [Fig. 3(a)]. Because of the low THz pulse energy, the energy change is quite small. However, the energy gain per unit of $\mathrm{THz}$ pulse energy is over 10 times higher than in the previous results, demonstrating the benefit of increased interaction lengths in the copropagating geometry. We achieve a THz-electron interaction distance of almost $15 \mathrm{~mm}$, which is around 5 times longer than that previously reported using single-cycle
THz pulses [16,20]. Compression of the bunch is shown in Fig. 3(b). At maximum compression, the electron bunch duration is reduced by a factor of 8 to a full width at half maximum of $180 \mathrm{fs}$ (FWHM), which is in agreement with the simulation performed with the particle-tracking program ASTRA [40], where around $1 \mathrm{fC}$ charge is used with a field gradient of $0.04 \mathrm{MV} / \mathrm{m}$. The FWHM pluses duration along the propagation direction is also shown in Fig. 4(e), black curve. A clear difference between compressed and uncompressed beams can be seen by the streaking deflectograms generated by plotting a lineout of the spatial charge distribution along the streaking dimension as a function of the delay relative to the THz field [Fig. 3(c)]. Focusing of the electron beam is shown in Fig. 3(d). At maximum focusing, the beam radius is reduced by a factor of 2.5 , and the peak intensity increased by approximately 6 times. The quality of the focused beam is similar to that produced by the conventional electromagnetic lens in front of the DLW.

\section{Cascaded DLW results}

In the single-DLW experiments, limited by the available $\mathrm{THz}$ energy, the initial velocity of the electrons is designed to match the phase velocity of the $\mathrm{THz}$, and the absolute average energy gain is sufficiently small, even for the acceleration experiments, that phase slippage is not the limiting factor. The limiting factor for the interaction length, in fact, is walk-off due to the large difference (a)

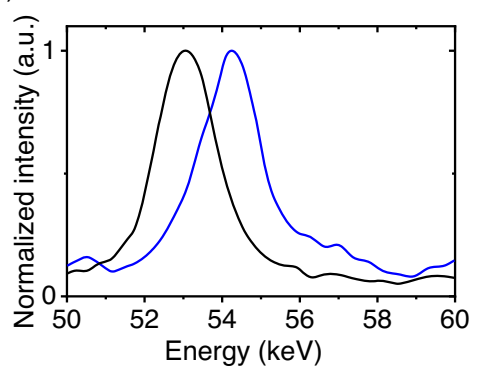

(b)

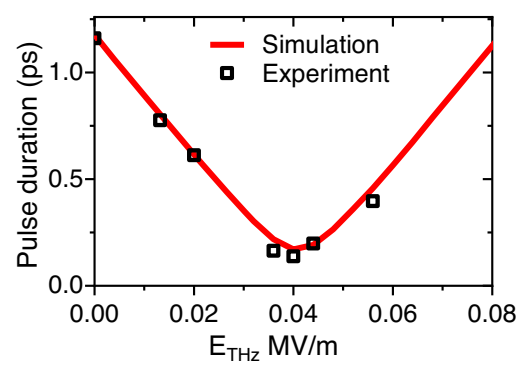

(c)

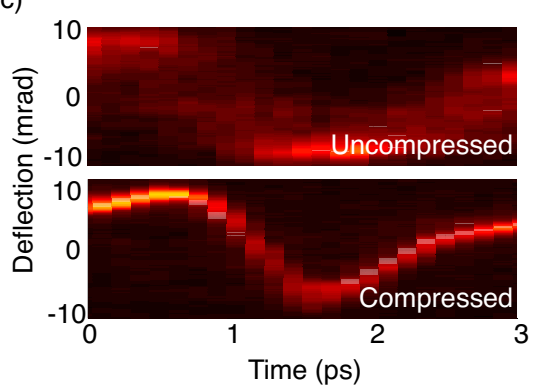

(d)

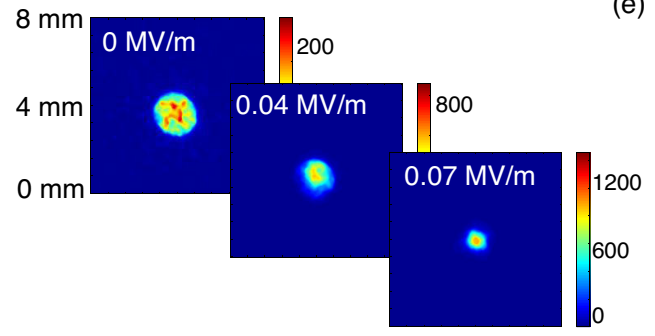

(e)

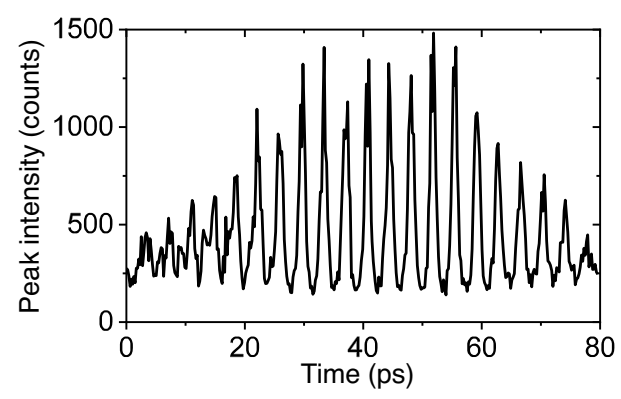

FIG. 3. DLW-based electron manipulation. (a) Measured electron energy spectra for the input beam (black curve) and accelerated beam (blue curve) in the acceleration mode. (b) Measured (hollow square) and simulated (red line) electron bunch length as a function of the applied THz field in compression mode. The simulation is performed with ASTRA with around $1 \mathrm{fC}$ charge injected into the DLW. (c) Time-dependent deflection diagrams measured by varying the delay between the arrival time of the electron bunch and the deflecting $\mathrm{THz}$ pulse for initially uncompressed and compressed electron bunches. (d) Electron beam diameter as a function of the THz field strength in focusing condition. (e) Electron peak intensity change as a function of the THz-electron delay. 
(a)

Terahertz lens

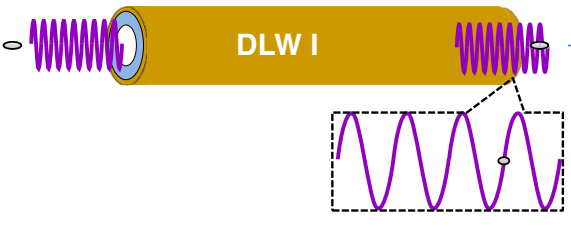

(b)

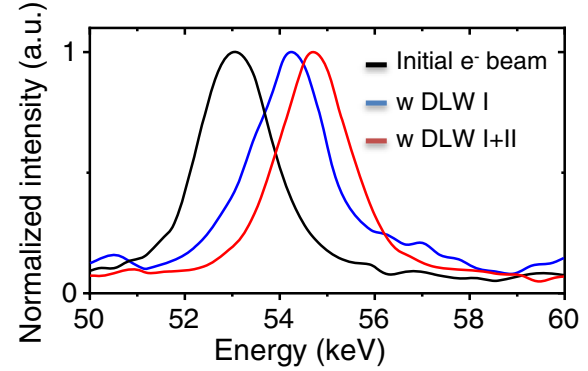

(d)

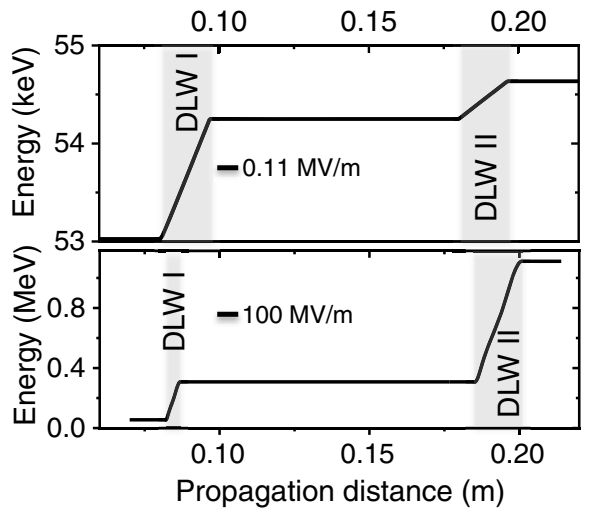

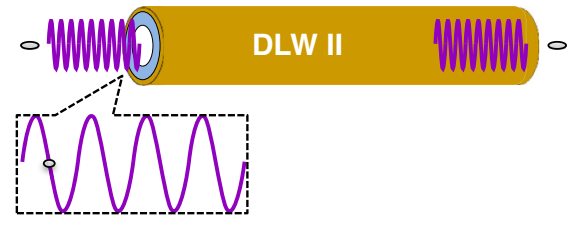

(c)

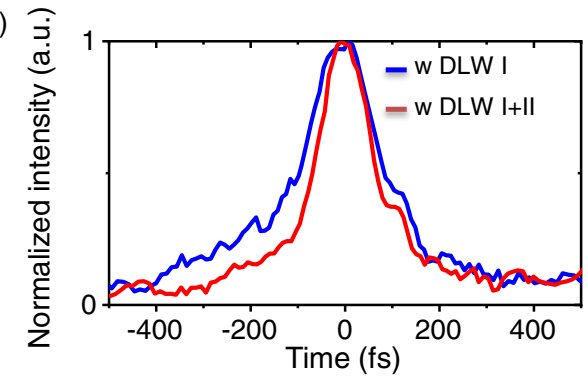

(e)

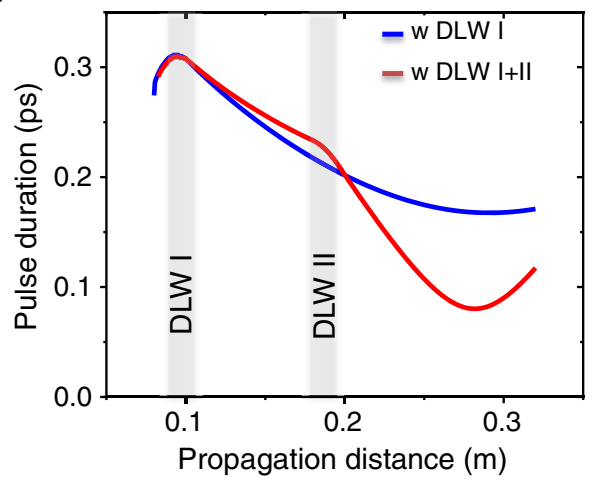

FIG. 4. Cascaded manipulation. (a) Schematic of the cascaded acceleration. Because of the low group velocity of the accelerating THz field inside the DLW, after THz-electron interaction, the electrons overtake the THz wave at the exit of DLW I. In a vacuum, the THz pulse catch up and overtake the electron bunch again before both enter DLW II for a second interaction, i.e., acceleration stage. (b) Measured electron energy spectra for the input beam (black curve), single-stage accelerated beam (blue curve), and cascaded electron acceleration (red curve). (c) Measured pulse duration via the single-stage compression (blue curve) and two-stage compression (red curve). (d) Top: Simulation of the experimental condition with two-stage cascaded acceleration that couples $20 \mathrm{~nJ}$ $\mathrm{THz}$ pulses with $0.11 \mathrm{MV} / \mathrm{m}$ longitudinal electric fields into the DLW I. The THz coupling efficiency from the first to second stage is approximately 10\%. Bottom: Simulation of two-stage cascaded acceleration with approximately $100 \mathrm{MV} / \mathrm{m}$ field gradients inside the DLW I and approximately $90 \%$ THz coupling from DLW I to DLW II. (e) Simulation of the electron pulse duration (FWHM) during propagation with a single DLW stage and two DLW stages. The coupling of THz into the second stage is assumed to be approximately $10 \%$. The simulation is performed with ASTRA with around $1 \mathrm{fC}$ charge injected into the DLW corresponding to the experimental conditions.

between the electron and pulse-envelope velocities. However, because the relative speed of the $\mathrm{THz}$ pulse and the electron bunch reverses outside the DLW, it is possible to recycle the $\mathrm{THz}$ pulses for a second interaction. As illustrated in Fig. 4(a), inside DLW I, the injected electrons travel faster than the envelope of the $\mathrm{THz}$ pulse and, therefore, overtake it. A horn coupler and a THz lens with a hole in the center are then used to couple $\mathrm{THz}$ into free space and collimate it while allowing the electrons to pass through. In a vacuum, the $\mathrm{THz}$ pulse has a higher group velocity than the electron bunch and, thus, catches up with and overtakes the electrons. During this propagation, the $\mathrm{THz}$ field has a negligible effect on the electrons due to the large size and resultant low field strength of the collimated $\mathrm{THz}$ beam. The $\mathrm{THz}$ radiation and electrons can then be coupled into a second DLW for a second interaction. The timing between the electrons and $\mathrm{THz}$ in the second DLW is set by controlling the distance between DLWs I and II using a motorized stage. Fine adjustments of the DLW II longitudinal position can also be used to select its operation mode. For example, to switch from focusing to defocusing requires shifting of the electrons by $180^{\circ}$ in phase relative to the $\mathrm{THz}$ waveform. Taking into account the speed of both the $\mathrm{THz}$ pulse and electron bunch, the 
required one cycle in position of DLW II is $\nu_{e} * \lambda /\left(c-\nu_{e}\right)=$ $0.856 \mathrm{~mm}$, which is less than the $\mathrm{THz}$ wavelength $(\lambda=1.153 \mathrm{~mm}$ at $260 \mathrm{GHz})$.

The results of the cascaded acceleration and compression experiments are shown in Fig. 4. By recycling the $\mathrm{THz}$ from DLW I, the electrons are further accelerated to $54.6 \mathrm{keV}$, nearly doubling the energy gain, which also agrees very well with the simulation [Fig. 4(d), top]. When electrons are accelerated using a fast-varying $\mathrm{THz}$ field, the bunch length needs to be much shorter than the duration of a $\mathrm{THz}$ half-cycle. This requirement is related to the variation of the electric field experienced by different electrons within the bunch arriving at different times. In general, a larger field gradient and longer bunch length lead to larger energy spreads [41]. For DLW I, the injected bunch length is approximately 300 fs (FWHM). For DLW II, a slight velocity bunching is performed in DLW I to enable a short electron bunch when injected into DLW II. Because of the small energy gain and limited energy resolution of the dipole spectrometer, no obvious energy spread is observed. However, the injected pulse duration can be especially important for high-energy acceleration, where a much shorter bunch length is desired to minimize the energy spread [41], which can be achieved with the same DLW in compression mode. Similarly, the cascaded compression results in a reduction of the bunch duration down to $150 \mathrm{fs}$ (FWHM), which is the limit of the streaker's time resolution. As can be seen from the simulations in Fig. 4(e), the actual bunch length is most likely shorter than is measured. This electron source can be already used for modern ultrafast electron diffraction experiments, where a short electron bunch with a high repetition rate is highly desired. If the longitudinal focus is too long, a single DLW I cannot compress the beam tightly (Fig. S4 [35]). Two DLWs provides high flexibility to control the beam parameter; for example, one can first increase the bunch length slightly with DLW I and then compress it to reach a shorter pulse duration at the target with DLW II. Because of the recycling of the $\mathrm{THz}$, the required energy for compression is reduced by around $10 \%$. By contrast, the observed improvement in focusing is not very large and is likely limited by the transverse emittance of the injected beam and long distance to the detector. Based on the acceleration results and the electron energy gain derived from simulations [Fig. 4(d), top], the $\mathrm{THz}$ coupling from the first to the second stage is estimated around $10 \%$. This coupling efficiency can be greatly improved by optimizing the $\mathrm{THz}$ telescope and horn coupler design, for example, adding coating on the lenses, using corrugation and curvature on the horn, and implementing a coupler to match the impedance. Even in the case when coupling efficiency is low, the low-energy $\mathrm{THz}$ in the second stage can also be used for other functions such as compression and focusing where a rather low field gradient is required.
We also simulate cascaded $\mathrm{THz}$ acceleration with around $100 \mathrm{MV} / \mathrm{m}$ longitudinal electric fields, which is well below the field breakdown thresholds in dielectric structures [31]. $55 \mathrm{keV}$ electrons can be accelerated to around $300 \mathrm{keV}$ in the first stage and beyond $1 \mathrm{MeV}$ in the second stage, with phase-velocity settings of $64 \%$ and $89 \%$ of the vacuum speed of light at $300 \mathrm{GHz}$ for DLW I and DLW II, respectively. Sub-mJ THz energy is required, which can be achieved with state-of-the-art THz technology [28]. With the increase of phase velocity, the $\mathrm{THz}$ energy is more concentrated in the center of the DLW. In the meantime, a smaller dimension can be used. Both result in a higher field gradient inside the DLW (see Supplemental Material [35] for detailed information). The dimension of the DLW should be optimized to provide a suitable phase and group velocity, which depends on the initial electron energy and THz field gradient inside the DLW. Because of the quick phase slippage under high-field-gradient conditions, DLW I must be kept very short or the timing between electron and $\mathrm{THz}$ pulse must be controlled in order to limit the interaction length and prevent dephasing. As can be seen from Fig. 4(d), the interaction length of an acceleration with $100 \mathrm{MV} / \mathrm{m} \mathrm{THz}$ fields is much shorter than with a lower field gradient. Higher fields lead to shorter interaction lengths, since the electron speed changes much faster. As the electron beam reaches relativistic speeds, it requires more drift time in free space for the THz pulse to overtake the electron bunch and, thus, a longer separation between DLWs I and II and longer travel distances for DLW II to switch between different operation modes. For example, it takes approximately $1690 \mathrm{~mm}$ for a $100-\mathrm{mm}$-long $\mathrm{THz}$ pulse to overtake a $1 \mathrm{MeV}(94 \% c)$ electron beam, and the relative cycle in DLW II is approximately $16 \mathrm{~mm}$. As the electron beam accelerates over successive stages, the interaction length must also increase, as can be seen in DLW II [Fig. 4(d), bottom].

\section{SUMMARY}

We demonstrate acceleration, temporal compression, and spatial focusing of fC charge electron bunches by using a cascaded DLW device powered with multicycle $\mathrm{THz}$ pulses. DLWs provide homogeneous spatial field distributions and are easy to implement. Taking advantage of the large group velocity difference, we are able to achieve the manipulation of electrons over centimeter-scale interaction lengths, which can be increased to even longer interaction distances by further recycling of the $\mathrm{THz}$ pulses via the cascaded manipulation scheme and, hence, lower $\mathrm{THz}$ energy required for the desired energy gain. This result is especially important, as the efficiency of current laserbased technologies for $\mathrm{THz}$ generation is still rather low $(<1 \%)$, which requires joule-level laser systems for $\mathrm{MeV}$ level electron acceleration. This cascading scheme will greatly lower the demand on the required laser system for electron acceleration in the nonrelativistic regime such as in 
the development of THz injector guns and ultrafast electron diffraction sources.

Currently, development of laser-based narrow-band $\mathrm{THz}$ generation is very intense and has resulted in the demonstration of $0.6 \mathrm{~mJ}$, subpercent-bandwidth $\mathrm{THz}$ pulses [28] which are ideal for copropagating THz-based acceleration. Numerical calculations predict that, with our scheme, sub$\mathrm{mJ} \mathrm{THz}$ energy is needed to reach the $\mathrm{MeV}$ threshold, which would represent a milestone achievement in $\mathrm{THz}$ acceleration. Schemes for reaching multi-mJ energies have also been described, are numerically analyzed, and are in the process of being tested $[42,43]$. It is therefore timely and pertinent to develop appropriate techniques for exploiting these upcoming powerful $\mathrm{THz}$ sources that can power multi-MeV electron sources that will truly be disruptive.

Multiple functions can be implemented using a single multicycle $\mathrm{THz}$ pulse by proper phasing of each stage. The physics insight provided by the cascaded manipulation scheme paves the way for further technology of practical implementation in applications from high-energy physics to ultrafast electron diffraction and tabletop x-ray sources.

\section{ACKNOWLEDGMENTS}

The authors gratefully acknowledge helpful discussion and technical support from Dr. Arya Fallahi, the expert support from Thomas Tilp and Matthias Schust for the fabrication of the DLW-device used in this work. We also acknowledge manufacturing of the magnetic steer for energy measurement by the group of Dr. Ralph Assmann at DESY. This work has been supported by the European Research Council under the European Union's Seventh Framework Program (FP7/2007-2013) through the Synergy Grant AXSIS (609920), the Cluster of Excellence "Advanced Imaging of Matter" of the Deutsche Forschungsgemeinschaft (DFG)-EXC 2056Project ID No. 390715994, and the accelerator on a chip program (ACHIP) funded by the Gordon and Betty Moore foundation (GBMF4744).

[1] G. Sciaini and R. J. D. Miller, Femtosecond Electron Diffraction: Heralding the Era of Atomically Resolved Dynamics, Rep. Prog. Phys. 74, 096101 (2011).

[2] S. Ideta, D. Zhang, A. G. Dijkstra, S. Artyukhin, S. Keskin, R. Cingolani, T. Shimojima, K. Ishizaka, H. Ishii, K. Kudo, M. Nohara, and R. J. D. Miller, Ultrafast Dissolution and Creation of Bonds in IrTe 2 Induced by Photodoping, Sci. Adv. 4, eaar3867 (2018).

[3] A. H. Zewail, 4D Ultrafast Electron Diffraction, Crystallography, and Microscopy, Annu. Rev. Phys. Chem. 57, 65 (2006).

[4] C. Bressler and M. Chergui, Ultrafast X-Ray Absorption Spectroscopy, Chem. Rev. 104, 1781 (2004).

[5] W. Leemans and E. Esarey, Laser-Driven Plasma-Wave Electron Accelerators, Phys. Today 62, No. 3, 44 (2009).
[6] V. Malka, J. Faure, Y. A. Gauduel, E. Lefebvre, A. Rousse, and K. T. Phuoc, Principles and Applications of Compact Laser-Plasma Accelerators, Nat. Phys. 4, 447 (2008).

[7] J. Breuer and P. Hommelhoff, Laser-Based Acceleration of Nonrelativistic Electrons at a Dielectric Structure, Phys. Rev. Lett. 111, 134803 (2013).

[8] E. A. Peralta, K. Soong, R. J. England, E. R. Colby, Z. Wu, B. Montazeri, C. McGuinness, J. McNeur, K. J. Leedle, D. Walz, E. B. Sozer, B. Cowan, B. Schwartz, G. Travish, and R. L. Byer, Demonstration of Electron Acceleration in a Laser-Driven Dielectric Microstructure, Nature (London) 503, 91 (2013).

[9] M. Dal Forno, V. Dolgashev, G. Bowden, C. Clarke, M. Hogan, D. McCormick, A. Novokhatski, B. Spataro, S. Weathersby, and S. G. Tantawi, Experimental Measurements of rf Breakdowns and Deflecting Gradients in mmWave Metallic Accelerating Structures, Phys. Rev. Accel. Beams 19, 051302 (2016).

[10] X. Wu, J. Shi, H. Chen, J. Shao, T. Abe, T. Higo, S. Matsumoto, and W. Wuensch, High-Gradient Breakdown Studies of an X-Band Compact Linear Collider Prototype Structure, Phys. Rev. Accel. Beams 20, 052001 (2017).

[11] J. Hebling, G. Almasi, I. Kozma, and J. Kuhl, Velocity Matching by Pulse Front Tilting for Large Area THz-Pulse Generation, Opt. Express 10, 1161 (2002).

[12] S.-W. Huang, E. Granados, W. R. Huang, K.-H. Hong, L. E. Zapata, and F. X. Kärtner, High Conversion Efficiency, High Energy Terahertz Pulses by Optical Rectification in Cryogenically Cooled Lithium Niobate, Opt. Lett. 38, 796 (2013).

[13] J. A. Fülöp, Z. Ollmann, C. Lombosi, C. Skrobol, S. Klingebiel, L. Pálfalvi, F. Krausz, S. Karsch, and J. Hebling, Efficient Generation of THz pulses with $0.4 \mathrm{~mJ}$ Energy, Opt. Express 22, 20155 (2014).

[14] C. Vicario, B. Monoszlai, and C. P. Hauri, GV/m Single-Cycle Terahertz Fields from a Laser-Driven Large-Size Partitioned Organic Crystal, Phys. Rev. Lett. 112, 213901 (2014).

[15] A. Fallahi, M. Fakhari, A. Yahaghi, M. Arrieta, and F. X. Kärtner, Short Electron Bunch Generation Using SingleCycle Ultrafast Electron Guns, Phys. Rev. Accel. Beams 19, 081302 (2016).

[16] E. A. Nanni, W. R. Huang, K.-H. Hong, K. Ravi, A. Fallahi, G. Moriena, R. J. D. Miller, and F. X. Kärtner, TerahertzDriven Linear Electron Acceleration, Nat. Commun. 6, 8486 (2015).

[17] D. A. Walsh, D. S. Lake, E. W. Snedden, M. J. Cliffe, D. M. Graham, and S. P. Jamison, Demonstration of Sub-luminal Propagation of Single-Cycle Terahertz Pulses for Particle Acceleration, Nat. Commun. 8, 421 (2017).

[18] C. Kealhofer, W. Schneider, D. Ehberger, A. Ryabov, F. Krausz, and P. Baum, All-Optical Control and Metrology of Electron Pulses, Science 352, 429 (2016).

[19] J. Fabiańska, G. Kassier, and T. Feurer, Split Ring Resonator Based THz-Driven Electron Streak Camera Featuring Femtosecond Resolution, Sci. Rep. 4, 5645 (2015).

[20] D. Zhang, A. Fallahi, M. Hemmer, X. Wu, M. Fakhari, Y. Hua, H. Cankaya, A.-L. Calendron, L. E. Zapata, N. H. Matlis, and F.X. Kärtner, Segmented Terahertz Electron Accelerator and Manipulator (STEAM), Nat. Photonics 12, 336 (2018). 
[21] S. Manz, A. Casandruc, D. Zhang, Y. Zhong, R. A. Loch, A. Marx, T. Hasegawa, L. C. Liu, S. Bayesteh, H. DelsimHashemi, M. Hoffmann, M. Felber, M. Hachmann, F. Mayet, J. Hirscht, S. Keskin, M. Hada, S. W. Epp, K. Flöttmann, and R. J. D. Miller, Mapping Atomic Motions with Ultrabright Electrons: Towards Fundamental Limits in Space-Time Resolution, Faraday Discuss. 177, 467 (2015).

[22] A. A. Ischenko, P. M. Weber, and R. J. D. Miller, Capturing Chemistry in Action with Electrons: Realization of Atomically Resolved Reaction Dynamics, Chem. Rev. 117, 11066 (2017).

[23] E. Curry, S. Fabbri, P. Musumeci, and A. Gover, THzDriven Zero-Slippage IFEL Scheme for Phase Space Manipulation, New J. Phys. 18, 113045 (2016).

[24] E. Curry, S. Fabbri, J. Maxson, P. Musumeci, and A. Gover, Meter-Scale Terahertz-Driven Acceleration of a Relativistic Beam, Phys. Rev. Lett. 120, 094801 (2018).

[25] L. J. Wong, A. Fallahi, and F. X. Kärtner, Compact Electron Acceleration and Bunch Compression in $\mathrm{THz}$ Waveguides, Opt. Express 21, 9792 (2013).

[26] A. L. Healy, G. Burt, and S. P. Jamison, Electron-Terahertz Interaction in Dielectric-Lined Waveguide Structures for Electron Manipulation, Nucl. Instrum. Methods Phys. Res., Sect. A, Detect. Assoc. Equip. 909, 199 (2018).

[27] Accu-Glass, https://www.accu-glass.com/.

[28] S. W. Jolly, N. H. Matlis, F. Ahr, V. Leroux, T. Eichner, A.-L. Calendron, H. Ishizuki, T. Taira, F. X. Kärtner, and A. R. Maier, Spectral Phase Control of Interfering Chirped Pulses for High-Energy Narrowband Terahertz Generation, Nat. Commun. 10, 2591 (2019).

[29] Y.-S. Lee, T. Meade, V. Perlin, H. Winful, T. B. Norris, and A. Galvanauskas, Generation of Narrow-Band Terahertz Radiation via Optical Rectification of Femtosecond Pulses in Periodically Poled Lithium Niobate, Appl. Phys. Lett. 76, 2505 (2000).

[30] F. Ahr, S. W. Jolly, N. H. Matlis, S. Carbajo, T. Kroh, K. Ravi, D. N. Schimpf, J. Schulte, H. Ishizuki, T. Taira, A. R. Maier, and F.X. Kärtner, Narrowband Terahertz Generation with Chirped-and-Delayed Laser Pulses in Periodically Poled Lithium Niobate, Opt. Lett. 42, 2118 (2017).

[31] M. C. Thompson, H. Badakov, A. M. Cook, J. B. Rosenzweig, R. Tikhoplav, G. Travish, I. Blumenfeld, M. J. Hogan, R. Ischebeck, N. Kirby, R. Siemann, D. Walz, P. Muggli, A. Scott, and R. B. Yoder, Breakdown Limits on Gigavolt-per-Meter
Electron-Beam-Driven Wakefields in Dielectric Structures, Phys. Rev. Lett. 100, 214801 (2008).

[32] F. Lemery, K. Floettmann, P. Piot, F. X. Kärtner, and R. Aßmann, Synchronous Acceleration with Tapered Dielectric-Lined Waveguides, Phys. Rev. Accel. Beams 21, 051302 (2018).

[33] A.-L. Calendron, H. Çankaya, and F. X. Kärtner, HighEnergy $\mathrm{kHz}$ Yb:KYW Dual-Crystal Regenerative Amplifier, Opt. Express 22, 24752 (2014).

[34] S. Carbajo, J. Schulte, X. Wu, K. Ravi, D. N. Schimpf, and F. X. Kärtner, Efficient Narrowband Terahertz Generation in Cryogenically Cooled Periodically Poled Lithium Niobate, Opt. Lett. 40, 5762 (2015).

[35] See Supplemental Material at http://link.aps.org/ supplemental/10.1103/PhysRevX.10.011067 for details of experimental methods, additional simulations and measurements.

[36] CST-Computer Simulation Technology, https://www.cst .com/.

[37] W. K. H. Panofsky and W. A. Wenzel, Some Considerations Concerning the Transverse Deflection of Charged Particles in Radio-Frequency Fields, Rev. Sci. Instrum. 27, 967 (1956).

[38] T. van Oudheusden, E. F. de Jong, S. B. van der Geer, W. P. E. M. O. 't Root, O. J. Luiten, and B. J. Siwick, Electron Source Concept for Single-Shot Sub-100 fs Electron Diffraction in the $100 \mathrm{keV}$ Range, J. Appl. Phys. 102, 093501 (2007).

[39] R. E. Collin, Foundations for Microwave Engineering (Wiley-IEEE Press, Hoboken, NJ, USA, 2001).

[40] K. Flöttmann, Astra-A space charge tracking algorithm, http://www.desy.de/ mpyflo.

[41] D. Zhang, A. Fallahi, M. Hemmer, H. Ye, M. Fakhari, Y. Hua, H. Cankaya, A.-L. Calendron, L. E. Zapata, N. H. Matlis, and F.X. Kärtner, Femtosecond Phase Control in High-Field Terahertz-Driven Ultrafast Electron Sources, Optica 6, 872 (2019).

[42] L. Wang, A. Fallahi, K. Ravi, and F. Kärtner, High Efficiency Terahertz Generation in a Multi-stage System, Opt. Express 26, 29744 (2018).

[43] D. N. Schimpf, H. T. Olgun, A. Kalaydzhyan, Y. Hua, N. H. Matlis, and F.X. Kärtner, Frequency-Comb-Based Laser System Producing Stable Optical Beat Pulses with Picosecond Durations Suitable for High-Precision Multi-cycle Terahertz-Wave Generation and Rapid Detection, Opt. Express 27, 11037 (2019). 